\title{
Obex Region
}

National Cancer Institute

\section{Source}

National Cancer Institute. Obex Region. NCI Thesaurus. Code C92592.

The location of the brain that corresponds to the narrowing of the fourth ventricle into the central canal of the spinal column. 